\title{
The Latest Results from the Durham/UKST Galaxy Redshift Survey
}

\author{
A. Broadbent, T. Shanks, R. Fong \\ Physics Dept., University of Durham, South Durham, Durham, UK \\ Q. A. Parker
}

Royal Observatory, Blackford Hill, Edinburgh, EHg 3HJ, UK

F. G. Watson

Royal Greenwich Observatory, Madingley Road, Cambridge, CBß OHA, $U K$

\begin{abstract}
We report on a redshift survey of $\sim 4000$ galaxies of B $\lesssim$ $17^{\mathrm{m}} 0$ covering a 1500 square degree area of sky around the SGP region. This 1-in-3 sampled survey probes a contiguous volume of $4 \times 10^{6} \mathrm{Mpc}^{3}$ to a depth of $300 h^{-1} \mathrm{Mpc}$. The survey has been compiled using the fibrelinked spectroscopy system, FLAIR, on the UK Schmidt Telescope and has produced high quality redshift maps giving a clear visual picture of structure in the universe as well as providing an excellent data base for a statistical analysis of galaxy clustering. Filamentary and sheet-like features are clearly discernible to the eye in the completed sections of the survey although the redshift two-point correlation function is consistent with zero on scales of $r \gtrless 10 h^{-1} \mathrm{Mpc}$. The survey, which is now substantially complete, demonstrates the potential of the FLAIR spectroscopy system for extending this project to a medium-deep galaxy redshift survey of the whole of the southern sky on timescales which would remain competitive with other surveys under construction.
\end{abstract}

\section{The Survey}

The 1-in-3 sample of galaxies is drawn from the Edinburgh/Durham Southern Galaxy Catalogue (Collins et al. 1988) which covers 60 UKST fields around the South Galactic Pole. The redshifts are obtained using FLAIR on the UK Schmidt Telescope in Australia which has two plate-holders with 92 and 73 $100 \mu \mathrm{m}$ fibre bundles. Typically $5 \times 3000 \mathrm{~s}$ exposures are required on any one field to obtain $\gtrsim 80 \%$ of the redshifts of galaxies of magnitude $B<17^{\mathrm{m}} 0$. With a plateholder change over time of only $40-60$ minutes, it is possible to obtain redshifts of $\sim 140$ galaxies in one night enabling the completion of such a large project on a time-scale which is competitive with other surveys currently under construction. Since $100 \mu \mathrm{m}$ fibres are used FLAIR can tolerate seeing conditions unsuitable for photographic survey work, making efficient use of the telescope. 


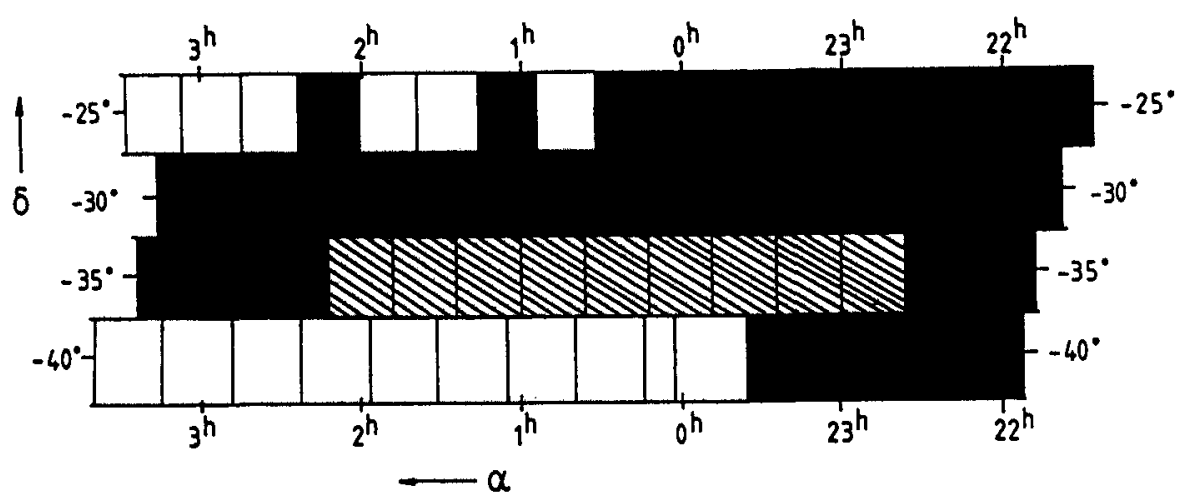

Figure 1. A plan of the Durham/UKST galaxy redshift survey area showing the individual field areas, those which have been observed (solid) and those which are part of the survey by Parker (hatched). The reduction and analysis described in this paper has been performed on galaxies which lie in the $\delta=-30^{\circ}$ strip between $21^{\mathrm{h}} 39$ to $02^{\mathrm{h}} 07$.

To date 36 of the 60 fields have been observed and a 1-in-3 sample has been drawn from the Parker redshift survey (Parker 1992) which covers 9 fields at $\delta=-35^{\circ}$ and has a similar magnitude limit (see Fig. 1). Twelve fields along a $60^{\circ} \times 5^{\circ}$ strip at $\delta=-30^{\circ}$ have been fully reduced and contains 544 galaxy redshifts with an accuracy of $\pm 150 \mathrm{kms}^{-1}$.

The cone plot in Fig. 2 represents the positions of the galaxies in RA and velocity. On inspection of this plot it can be seen that there are apparently large structures in the distribution on scales of $\sim 100 h^{-1} \mathrm{Mpc}$. Among the most noticeable of these are the long filamentary structures surrounding a region of low density between 0 and $8000 \mathrm{kms}^{-1}$ which has been referred to as the Sculptor void (Fairall \& Jones 1988). Structures are discernible at higher redshifts, in particular, the possible wall structure at $\sim 18000 \mathrm{kms}^{-1}$. Not surprisingly, the $\mathrm{n}(\mathrm{z})$ histogram plot in Fig. 3 has two strong peaks at 8000 and $18000 \mathrm{kms}^{-1}$. There also is evidence of similar structures in the sample taken from the survey of Parker.

\section{Analysis}

We have made a statistical investigation of the nature of the galaxy distribution in the RA slice at $\delta=-30^{\circ}$ by calculating the redshift two-point correlation function, $\xi_{s}(s)$. This is a measure in velocity space of the excess probability of finding a galaxy in a volume element over a random and homogeneous distribution and was calculated using the methods of Shanks et al. (1983). On comparison with previous surveys (Durham/AAT, Durham/SAAO and pilot FLAIR surveys, Peterson et al. 1986; Metcalfe et al. 1989; Watson et al. 1991) the $\xi_{s}(s)$ thus derived shows reasonable agreement within the errors. 


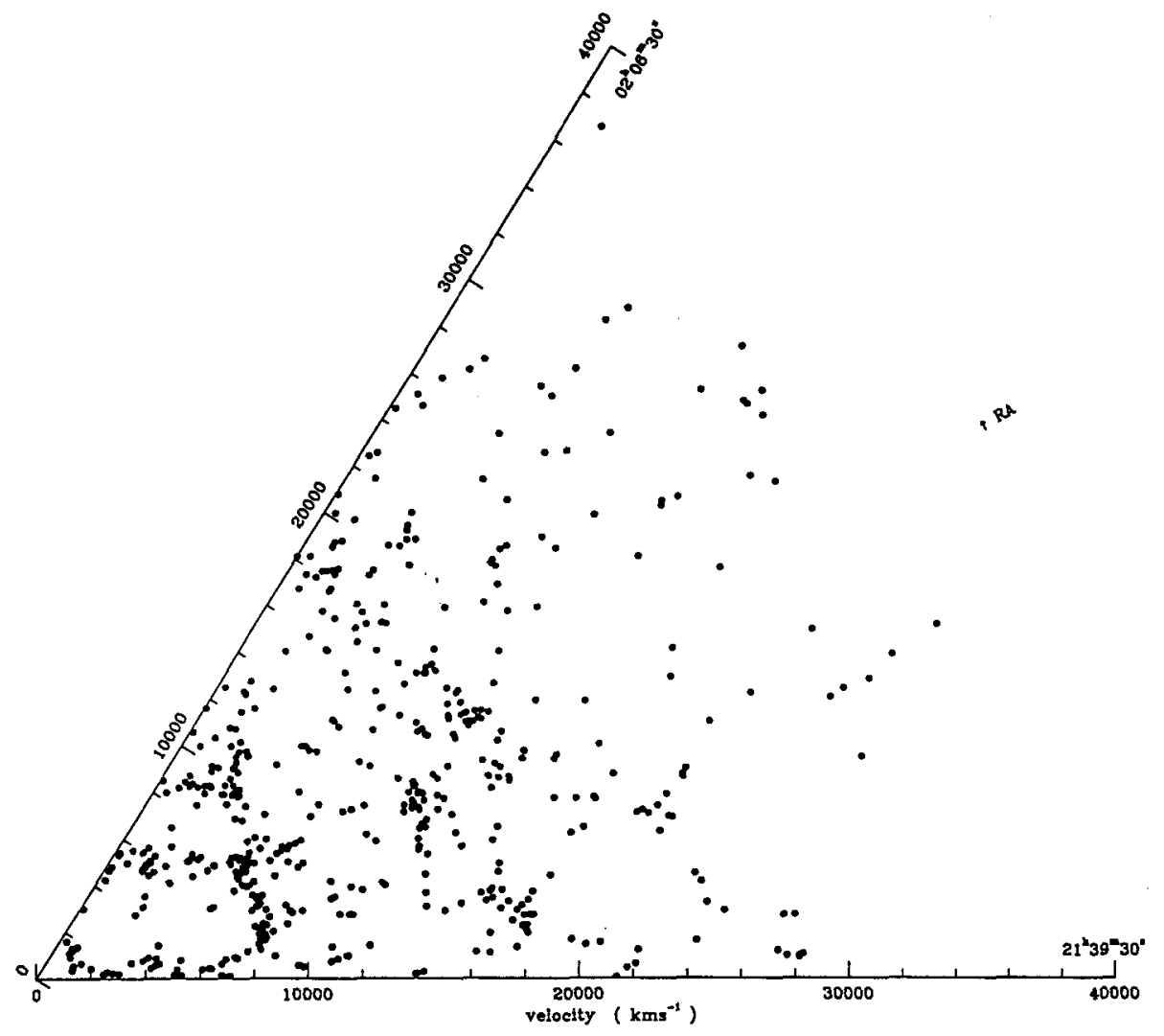

Figure 2. A cone-plot of the galaxy distribution in a $60^{\circ} \mathrm{RA}$ strip at $\delta=-30^{\circ}$ from the Durham/ UKST redshift survey. Redshift increases across the page and RA increases up the page. Strong filamentary clustering on scales of $\sim 100 h^{-1} \mathrm{Mpc}$ can be seen along and across the line of sight. 


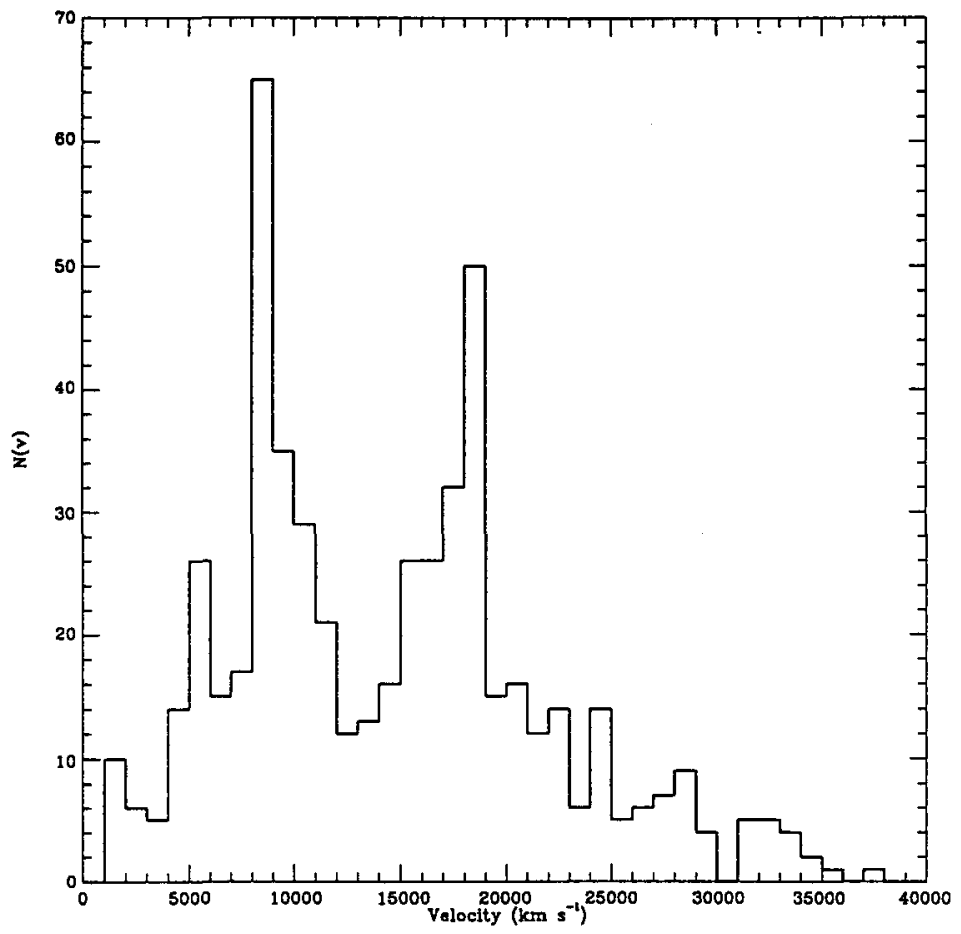

Figure 3. A redshift histogram of the galaxies in the Durham/UKST survey. There are two prominent peaks at $\sim 8000$ and $\sim 1800 \mathrm{kms}^{-1}$. Again, the galaxy distribution looks inhomogeneous on scales of $100 h^{-1} \mathrm{Mpc}$. 


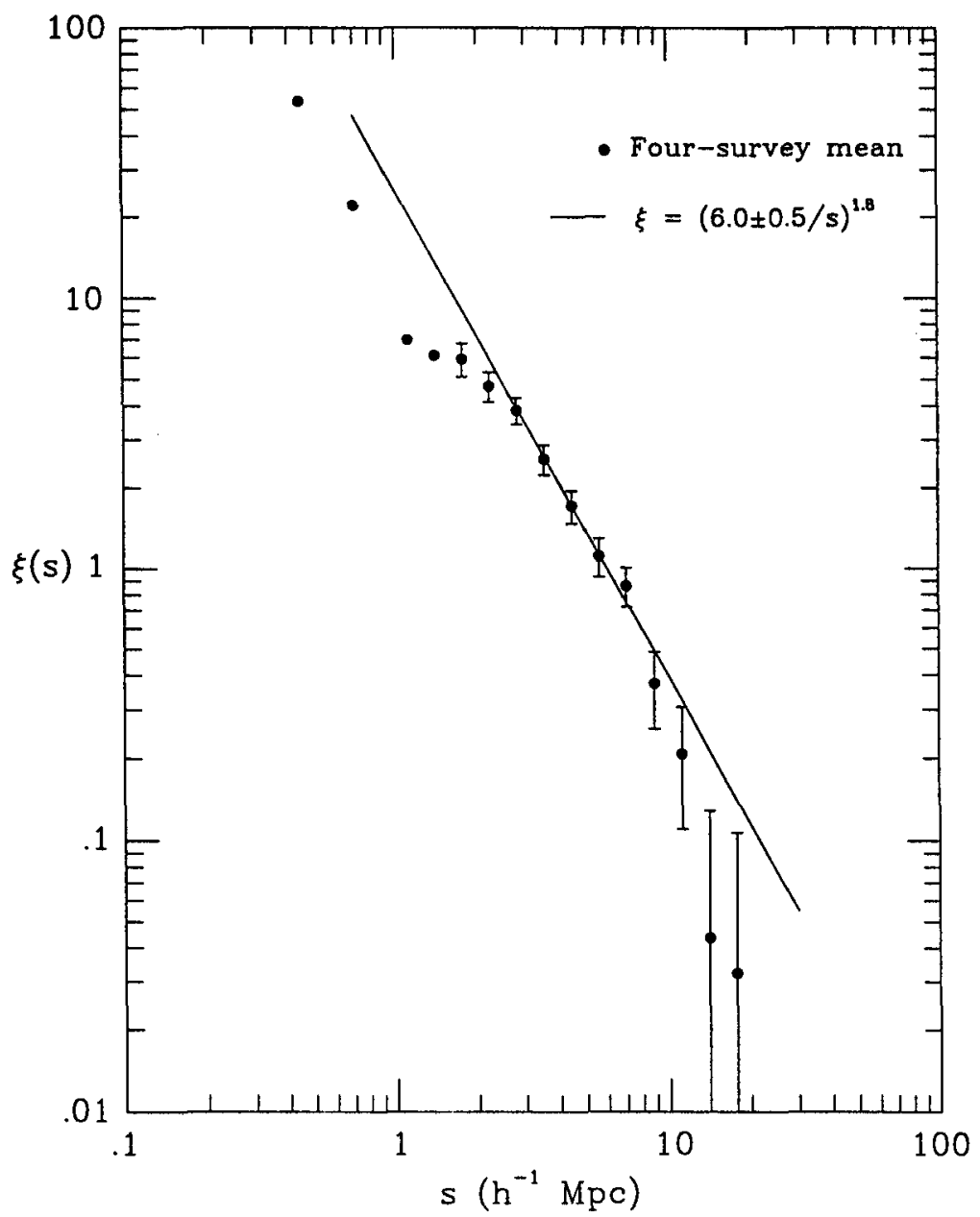

Figure 4. The average small scale redshift two-point correlation function from the Durham/UKST, Durham/AAT, Durham/SAAO and pilot FLAIR galaxy redshift surveys. The solid line is a 1.8 power law approximation to the form of $\xi_{s}$ between 2 and $7 h^{-1} \mathrm{Mpc}$. The error on the amplitude is estimated from the survey-to-survey variation. 


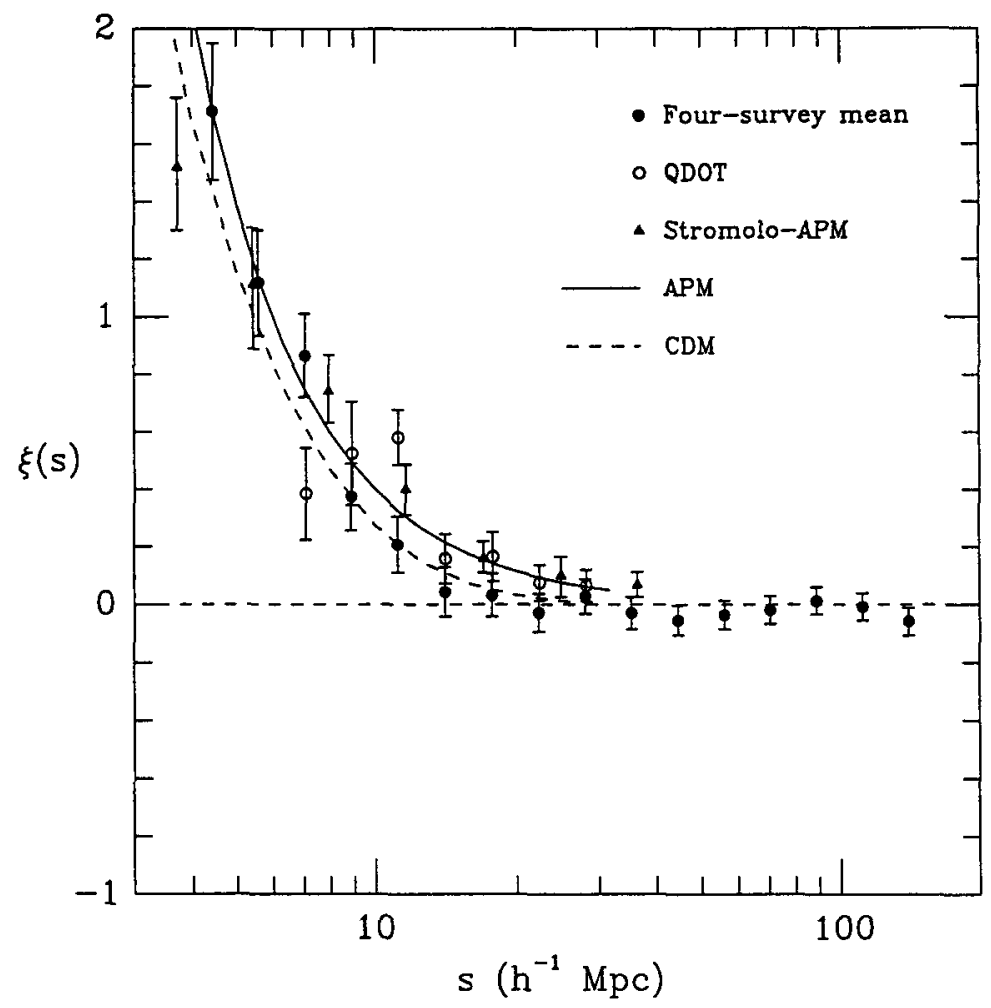

Figure 5. The average redshift two-point correlation function at large scales derived from galaxies in the Durham/UKST redshift survey, the Durham/AAT, Durham/SAAO and pilot FLAIR surveys. The open circles represent the correlation function calculated from IRAS galaxies and the triangles that from the APM-Stromolo redshift survey. The solid line is an approximation to $\xi$ derived from the APM angular twopoint correlation function, $\omega(\theta)$ and the dashed line is the predicted $\xi$ from standard CDM theory. Our results show no significant power beyond $10 h^{-1} \mathrm{Mpc}$ although we cannot rule out the APM result at more than the $2 \sigma$ level. 
The results of all four surveys are readily combined to produce an error weighted mean which effectively samples 1300 galaxy redshifts within a volume of $\sim 2 \times 10^{6} \mathrm{Mpc}^{3}$. The resulting mean $\xi_{s}$ is shown on large and small scales in Figs 4 and 5 respectively.

The straight line in Fig. 4 is a power law approximation to $\xi_{s}$ between 2 and $7 h^{-1} \mathrm{Mpc}$. The deviation from a pure power law between 1 and $2 h^{-1} \mathrm{Mpc}$ was remarked upon in the AAT and SAAO surveys alone and an analysis of the correlation function in directions along and perpendicular to the line of sight should help to clarify whether this is due to peculiar motion and infall or true, non-scale-free clustering of galaxies on small scales.

On larger scales $>10 h^{-1} \mathrm{Mpc}$ (Fig. 5), the points are consistent with zero correlation at the $1 \sigma$ level despite the large scale features seen in the cone plot in Fig. 2. Other analyses not relying on the second moment of the distribution will be required to define the filamentary structures. In Fig. 5 we compare our results with those from two other large redshift surveys. The $\xi$ calculated from the IRAS-QDOT survey (Saunders et al. 1992) shows a tendency to be higher than our own on scales of $8-30 h^{-1} \mathrm{Mpc}$ as does that from the APM-Stromlo redshift survey (Loveday et al. 1992) although the discrepancies between the surveys are only at the $\sim 1 \sigma$ level.

The solid curve in Fig. 5 is an estimate of $\xi$ derived from the angular two-point correlation function $(\omega(\theta))$ of the APM survey (Maddox et al. 1990) and the dashed line that predicted from a standard biased CDM model with $\Omega=1$ calculated using the formulation of Hamilton et al. 1991. The APM $\omega(\theta)$ apparently detects clustering above that expected by standard CDM theory to a high level of confidence although there is always the possibility of small systematic errors confusing the correlation function on larger scales. The redshift two-point correlation function however is less sensitive to such effects.

\section{Future}

The Durham/UKST galaxy redshift survey should be completed within the next year and the results of the reduced sections demonstrate the potential of the whole survey as a tool for testing theoretical models such as CDM in addition to providing a clearer visual picture of large scale structure in the Universe. We have demonstated that the FLAIR system is an efficient and competitive means of gathering large numbers of galaxy redshifts and would envisage extending the current survey to produce a medium-deep suvey of the whole of the southern sky.

\section{References}

Collins C. A., Heydon-Dumbleton N. H. \& MacGillivray H. T., 1988, MNRAS, 236,7

Fairall A. P. \& Jones A., 1988, Publs. Dept. Astr. Cape Town, 10

Hamilton A. J. S., Kumar P., Lu, E. \& Matthews A., 1991, ApJ, 374, L1

Loveday J., Efstathiou G., Peterson B. A. \& Maddox S. J., 1992, ApJ, 400, L43

Maddox S. J., Efstathiou G. P. \& Sutherland W. J., 1990, MNRAS, 246, 433 
Metcalfe N., Fong R., Shanks T. \& Kilkenny D., 1989, MNRAS, 236, 207

Parker Q. A., 1992, in Digitised Optical Sky Surveys, H. T. MacGillivray \& E. B. Thomson, (Kluwer), p.383

Peterson B. A., Ellis R. S., Efstathiou G. P., Shanks T., Bean A. J., Fong R. \& Zen-Long Z., 1986, MNRAS, 221, 233

Saunders W., Rowan-Robinson M. \& Lawrence A., 1992, MNRAS, 258, 134

Shanks T., Bean A. J., Efstathiou G. P., Ellis R. S., Fong R. \& Peterson B. A., 1983, ApJ, 274, 529

Watson F. G., Oates A. P., Shanks T. \& Hale-Sutton D., 1991, MNRAS, 253, 222

\section{Discussion}

Tsvetkov: Can you make some comparison between your survey and the Muenster redshift survey, with UKST observational material?

Broadbent: The Muenster redshift survey to which you refer is based on crude estimates from low dispersion objective-prism plates where the redshift 'accuracy' is claimed to be $\pm 3000 \mathrm{~km} \mathrm{~s}^{-1}$. The detailed topological detail evident in our survey (voids, filaments, sheets etc.) are not clearly seen in the Muenster results. 